\title{
Magnetic resonance imaging, radiography, and scintigraphy of the finger joints: one year follow up of patients with early arthritis
}

M Klarlund, M Østergaard, K E Jensen, J Lysgård Madsen, H Skjødt, I Lorenzen, and the TIRA group ${ }^{\star}$
The Danish Research Centre of Magnetic Resonance, H:S Hvidovre Hospital, University of Copenhagen M Klarlund M Østergaard $\mathrm{K}$ E Jensen

Department Internal Medicine, Division of Rheumatology, H:S Hvidovre Hospital M Klarlund

M Østergaard

H Skjødt

I Lorenzen

Department of Radiology, H:S

Hvidovre Hospital K E Jensen

Department of Clinical Physiology and Nuclear Medicine, H:S Hvidovre Hospital J Lysgård Madsen

*TIRA stands (in Danish) for early rheumatoid arthritis. Others from the TIRA group were: Julia Sidenius Johansen, Trine Jensen, Merete Hetland, and Søren Jacobsen

Correspondence to: Dr Mette Klarlund, Department of Interna Medicine, Division of Rheumatology, H:S Hvidovre Hospital, Kettegaard Allé 30, DK-2650 Hvidovre, Denmark

Email: klarlund@dadlnet.dk

Accepted for publication 3 February 2000

\begin{abstract}
Objectives-To evaluate synovial membrane hypertrophy, tenosynovitis, and erosion development of the 2 nd to 5 th metacarpophalangeal (MCP) and proximal interphalangeal (PIP) joints by magnetic resonance imaging in a group of patients with rheumatoid arthritis (RA) or suspected RA followed up for one year. Additionally, to compare the results with radiography, bone scintigraphy, and clinical findings.

Patients and methods-Fifty five patients were examined at baseline, of whom 34 were followed up for one year. Twenty one patients already fulfilled the American College of Rheumatology (ACR) criteria for RA at baseline, five fulfilled the criteria only after one year's follow up, whereas eight maintained the original diagnosis of early unclassified polyarthritis. The following MRI variables were assessed at baseline and one year: synovial membrane hypertrophy score, number of erosions, and tenosynovitis score.

Results-MRI detected progression of erosions earlier and more often than did radiography of the same joints; at baseline the MRI to radiography ratio was 28:4. Erosions were exclusively found in patients with RA at baseline or fulfilling the ACR criteria at one year. At one year follow up, scores of MR synovial membrane hypertrophy, tenosynovitis, and scintigraphic tracer accumulation had not changed significantly from baseline; in contrast, swollen and tender joint counts had declined significantly $(p<0.05)$.

Conclusions-MRI detected more erosions than radiography. MR synovial membrane hypertrophy and scintigraphy scores did not parallel the changes seen over time in clinically assessed swollen and tender joint counts. Although joint disease activity may be assessed as quiescent by conventional clinical methods, a more detailed evaluation by MRI may show that a pathological condition is still present within the synovium.

(Ann Rheum Dis 2000;59:521-528)
\end{abstract}

The joints of the hands are among the first to be affected in rheumatoid arthritis (RA) $)^{1-3}$ and are therefore of particular interest in the assessment of patients with suspected early RA. Early intervention with disease modifying antirheumatic drugs (DMARDs) in RA to prevent progression of inflammatory and destructive changes in the joints requires that it is possible to distinguish between severe and mild self limiting cases in the early stages of disease. ${ }^{4}$ Magnetic resonance imaging (MRI) has been shown to be a more sensitive method than radiography to demonstrate inflammatory and destructive changes in the knee and wrist joints of patients with RA. ${ }^{5-9}$ However, imaging of the finger joints demands intense computations so far limited to specially designed research equipment. Cross sectional studies on clinical MRI units have shown the feasibility of MRI of the hand $\mathrm{d}^{10-12}$ and quantification of synovium in the wrist or the whole hand. ${ }^{13-15}$ Cross sectional studies of the finger joints in RA have been undertaken which applied qualitative methods, ${ }^{16-19}$ and, recently, quantitative methods have also been used. ${ }^{2021}$

Few longitudinal studies have been undertaken in this field..$^{15}{ }^{22} 23$ In these studies all the patients met the American College of Rheumatology (ACR) criteria for RA and had had a long disease duration at entry; in one study median duration was 122 months $^{22}$ and in another it was five years on average $\mathrm{e}^{15}$; a third study focused on joints in which erosive changes on plain films had been found at entry to the study. ${ }^{16}$ All these studies mainly described changes in the synovium and compared these findings with radiological findings. A single case study of one patient showed progression of erosion in a finger joint of a patient with RA despite treatment. ${ }^{23}$

We have followed up patients with early arthritis symptoms prone to develop RA and assessed inflammatory and destructive changes in the finger joints by MRI. To our knowledge this has not been described before. Because destructive changes in RA joints have been shown to occur most rapidly during the first years of disease ${ }^{24-27}$ we report our findings after one year's follow up. The course of synovial membrane hypertrophy, tenosynovitis, and erosion development of the 2nd to 5th metacarpophalangeal (MCP) joints and proximal interphalangeal (PIP) joints was followed by MRI, and the results were compared with conventional radiography, bone scintigraphy, and clinical findings.

\section{Patients and methods}

PATIENTS

Eligible for the study were patients who had symmetrically swollen or tender 2 nd or 3rd MCP or PIP joints, who had had symptoms for 
fewer than two years, and who were willing to undergo repeated examinations by a doctor, MRI, scintigraphy, and radiography.

The report presents data obtained at baseline and at one year follow up. At baseline, 55 patients were examined by MRI and radiography. At one year, 34 of these were examined again by MRI. The reasons for dropping out of the study were the following: patient died (two), moved to another area (one), became pregnant (two), felt uncomfortable during the contrast injection (one), could not stand the MRI examination owing to claustrophobia or asthma bronchialis (six), or were not interested in continuing (nine).

Owing to limited capacity for scintigraphy, only 24 of the 55 patients were examined at baseline by scintigraphy. Fourteen patients were examined again at one year.

\section{CLINICAL ASSESSMENTS}

Clinical examination of each patient was carried out by the same rheumatologist at baseline and at one year. Data collected at baseline and at one year included swollen joint count, tender joint count, doctor's global assessment, patient's global assessment, and Health Assessment Questionnaire score (HAQ score, functional disability score) according to the core set of disease activity measures as defined by the ACR, ${ }^{28} 29$ and erythrocyte sedimentation rate (normal level $<20 \mathrm{~mm} / 1$ st $\mathrm{h}$ ), serum $\mathrm{C}$ reactive protein (normal level $<95 \mathrm{nmol} / \mathrm{l}$ ), and rheumatoid factor (IgM RF, assessed by nepholometry). For comparison with MRI, swelling (defined as palpable synovial thickening) and tenderness in each of the 2 nd to 5 th MCP and PIP joints were noted separately.

The patients were treated after a protocol aimed at suppressing inflammation with nonsteroidal anti-inflammatory drugs (NSAIDs), DMARDs (methotrexate or sulfasalazine) or a combination of DMARDs, NSAIDs, and corticosteroids. As shown in table 1 some of the patients with RA had mild disease and refrained from receiving DMARD treatment.

The study was conducted in accordance with the Declaration of Helsinki, and the protocol was approved by the local ethics committee. Signed informed consent was obtained from all subjects. Other data from this study cohort will be published elsewhere.

\section{MRI ASSESSMENT}

MRI of the 2nd to 5th MCP joints of the dominant hand was obtained at baseline and at the one year follow up visit on a $1.0 \mathrm{~T}$ Siemens Magnetom Impact Unit (Erlangen, Germany) equipped with a receive only, wrap around surface coil. The patients were placed supine with the arm along the side of the body and the hand fixed sagittally with a specially designed splint to avoid movement. Continuous axial and coronal, T1 weighted, spin echo images of the hand (TR/TE/slice thickness: $600-700 \mathrm{~ms} / 15$ $\mathrm{ms} / 3 \mathrm{~mm}$ ) were obtained before and after intravenous injection of $0.1 \mathrm{mmol} / \mathrm{kg}$ body weight of gadolinium-DTPA (Gd-DTPA, Magnevist, Schering, Berlin, Germany). The matrix size was $224 \times 256$ and the field of view $120 \mathrm{~mm}$.

MR scoring of synovial membrane hypertrophy was done semiquantitatively by comparing before and after contrast axial and coronal T1 weighted SE images while displayed on the terminal. Each joint was evaluated on a progressive scale from 0 to 4 as follows: $0=$ no visible enhancement; 1 = only slight enhancement; 2 $=$ marked enhancement of small areas; $3=$ marked enhancement of moderately sized areas; $4=$ marked enhancement of large areas; see reference ${ }^{19}$ for details. The sum of scores for the eight joints resulted in values ranging from 0 to 32 for each patient. Tenosynovitis was scored for the presence or absence of enhancement in the tendon sheets (sum of scores, range $0-8)$. MR erosions had to be visible on both axial and coronal slices to be diagnosed. The

Table 1 Baseline and one year follow up characteristics of patients

\begin{tabular}{|c|c|c|c|c|c|c|c|c|}
\hline & \multirow{2}{*}{$\begin{array}{l}\text { All patients, } \\
\text { baseline } \\
(n=55)\end{array}$} & \multirow{2}{*}{$\begin{array}{l}\text { Patients in the } \\
\text { one year follow } \\
\text { up study, } \\
\text { baseline }(n=34)\end{array}$} & \multicolumn{2}{|l|}{$R A$ group } & \multicolumn{2}{|l|}{ New-RA group } & \multicolumn{2}{|l|}{$P A^{\star}$ group } \\
\hline & & & $\begin{array}{l}\text { Baseline } \\
(n=21)\end{array}$ & 1 Year & $\begin{array}{l}\text { Baseline } \\
(n=5)\end{array}$ & 1 Year & $\begin{array}{l}\text { Baseline } \\
(n=8)\end{array}$ & 1 Year \\
\hline \multicolumn{9}{|l|}{ Demographic } \\
\hline Sex (female/male) & $42 / 13$ & $23 / 11$ & $11 / 10$ & & $5 / 0$ & & $7 / 1$ & \\
\hline Age (years) & $51(20-82)$ & $50(20-82)$ & $55(29-82)$ & & $40(20-56)$ & & $40(13-68)$ & \\
\hline Disease duration (months) & $4(0-24)$ & $3(0-22)$ & $3(0-22)$ & & $3(2-5)$ & & $3.5(1-13)$ & \\
\hline IgM RF* (No (\%) positive) & $27(49)$ & $16(47)$ & $13(62)$ & & $2(40)$ & & $2(25)$ & \\
\hline \multicolumn{9}{|l|}{ Drug treatment $\dagger$} \\
\hline NSAIDs* ${ }^{\star}$ & $48(87)$ & $23(68)$ & $19(90)$ & $5(24)$ & $1(20)$ & $4(80)$ & $3(38)$ & $1(13)$ \\
\hline DMARDs* & $14(25)$ & $8(24)$ & $8(38)$ & $14(67)$ & 0 & $3(60)$ & $1(13)$ & \\
\hline Steroids & $17(31)$ & $10(29)$ & $8(38)$ & $3(14)$ & 0 & 0 & $2(25)$ & 0 \\
\hline \multicolumn{9}{|l|}{ Measures of disease activity $\neq$} \\
\hline Swollen joint count & $5(0-18)$ & $6(0-18)$ & $6(0-18)$ & $0(0-6)$ & $3(0-8)$ & $2(0-4)$ & $4(0-11)$ & $0(0-0)$ \\
\hline Tender joint count & $16(0-24)$ & $15(0-24)$ & $16(0-24)$ & $0(0-16)$ & $13(8-20)$ & $2(0-24)$ & $15(5-24)$ & $0(0-24)$ \\
\hline Patient global assessment & $40(0-88)$ & $44(0-82)$ & $44(4-78)$ & $10(0-65)$ & $23(11-53)$ & $12(0-68)$ & $30(0-82)$ & $8(0-70)$ \\
\hline Doctor global assessment & $18(0-79)$ & $18(0-79)$ & $28(0-79)$ & $2(0-42)$ & $11(3-35)$ & $18(0-25)$ & $15(3-39)$ & $2(0-3)$ \\
\hline Pain & $33(0-84)$ & $34(0-74)$ & $41(4-74)$ & $7(0-56)$ & $24(14-51)$ & $14(0-65)$ & $16(0-71)$ & $4(0-68)$ \\
\hline $\mathrm{HAQ}^{\star}$ score & $0.75(0-2)$ & $0.75(0-2)$ & $0.8(0-2)$ & $0.1(0-1.3)$ & $0.5(0.4-1.13)$ & $0(0-1.8)$ & $0.5(0-2)$ & $0.1(0-1.3)$ \\
\hline $\mathrm{ESR}^{\star}(\mathrm{mm} / 1 \mathrm{st} \mathrm{h})$ & $20(3-105)$ & $15(3-42)$ & $20(3-42)$ & $14(2-105)$ & $14(3-24)$ & $12(3-32)$ & $7(4-12)$ & $4(0-12)$ \\
\hline Serum $\mathrm{CRP}^{\star}(\mathrm{nmol} / \mathrm{l})$ ฯ & $95(95-1365)$ & $95(95-543)$ & $116(95-543)$ & $95(95-1027)$ & $95(95-95)$ & $95(95-143)$ & $95(95-95)$ & $95(95-95)$ \\
\hline
\end{tabular}

^PA = early unclassified polyarthritis; $\mathrm{RF}=$ rheumatoid factor; NSAIDs = non-steroidal anti-inflammatory drugs; DMARDs = disease modifying antirheumatic drugs; $\mathrm{HAQ}=$ Health Assessment Questionnaire; ESR = erythrocyte sedimentation rate; s-CRP = serum C reactive protein

tNo (\%) of patients receiving NSAIDs, DMARDs, and steroids.

$\ddagger$ Values are median (range).

IRaised when $>95 \mathrm{nmol} / \mathrm{l}$. 


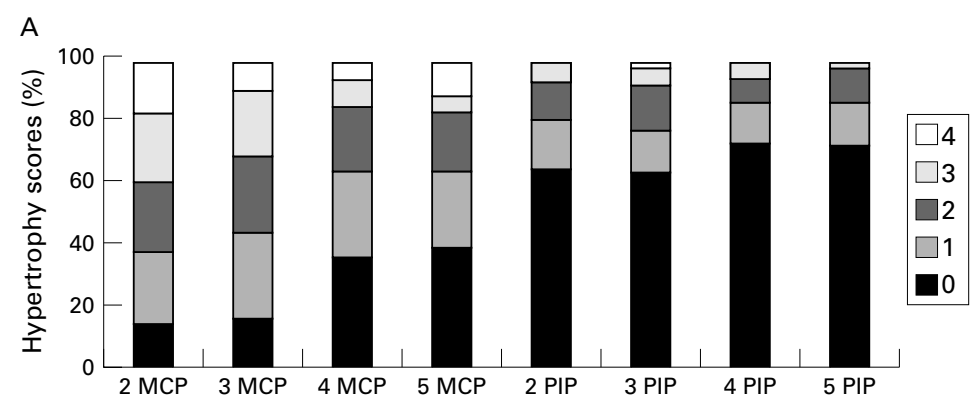

B

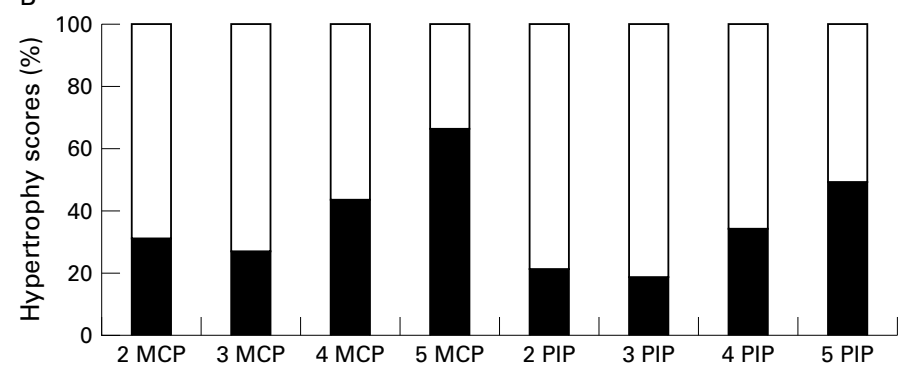

Figure 1 (A) Distribution of magnetic resonance imaging synovial membrane hypertrophy scores (0-4) in each finger joint (given as a percentage for each score (0-4)). $(B)$ Clinical assessment in individual 2 nd to 5 th metacarpophalangeal (MCP) and proximal interphalangeal (PIP) joints at baseline (55 patients); black indicates the percentage of joints without swelling and tenderness while white indicates the percentage of joints with swelling or tenderness, or both.

number of MR erosions in each finger joint was counted (at baseline and at the one year follow up). The position of each erosion was marked on a drawing of the hand. Enlargement of MR erosions at the follow up was judged by comparing erosions seen at baseline with the erosions seen at follow up with both pictures simultaneously on the screen. If it seemed helpful for the review, diameters of the erosions were measured. MR images were presented in an ordered fashion (baseline, one year) ${ }^{30}$ and were evaluated blinded (by $\mathrm{MK}$ ) to clinical, radiographic, and scintigraphic findings.

RADIOGRAPHIC ASSESSMENT

Conventional radiography was obtained of both hands and wrists in the posterior-anterior and Nørgaard views ${ }^{31}$ at baseline and at the one year follow up.

All radiological assessments were performed by the same radiologist (KEJ) blinded to clinical, MRI, and scintigraphic findings. Radiographs were presented in an ordered fashion. The number of erosions and possible enlargement of the erosions at the follow up in each finger joint and wrist was scored. The position of the erosions was marked on a drawing of the hand. Additionally, radiographs of both hands and wrists were evaluated according to the Larsen method. ${ }^{32} 33$

SCINTIGRAPHIC ASSESSMENT

Scintigraphy of both hands corresponding to the bone phase was performed using a StarrCam XR/7 gammacamera equipped with a low energy resolution collimator (GE Medical Systems, Milwaukee, Ill, USA), three to four hours after injection of $550-650 \mathrm{MBq}$ of the radionuclide technetium-99m labelled methylene diphosphonate. A palmar projection of the hands was used. Details of the 2 nd to 5 th MCP and PIP joints of the dominant hand were evaluated for comparison with the MRI findings. Each finger joint was evaluated on a scale from 0 to 3 according to the degree of tracer accumulation: $0=$ negative; $1=$ mildly positive $;=$ positive $; 3=$ strongly positive (sum of scores, range 0-24). Scintigrams were read in an ordered fashion (by JLM), blinded to other findings.

\section{STATISTICS}

The Mann-Whitney U test (two sample rank sum test) was used for comparison between groups. The Wilcoxon matched (one sample signed rank sum test) pairs test was used to test changes after one year.

\section{Results}

At entry, 31 of 55 patients fulfilled the ACR 1987 classification criteria, ${ }^{34}$ while 24 patients did not. Table 1 gives details of other baseline characteristics of the 55 patients.

Baseline and follow up characteristics of the group of 34 patients followed up for one year are also presented in table $1 ; 21$ fulfilled the ACR 1987 criteria at baseline (RA group), eight patients were classified as early undifferentiated polyarthritis (PA group) and maintained this diagnosis, whereas five patients progressed during the one year observation period and fulfilled the ACR 1987 classification criteria only at the one year follow up (new-RA group) (table 1).

MRI OF THE 2ND TO 5TH MCP AND PIP JOINTS OF DOMINANT HANDS

Baseline findings for all patients $(n=55)$

Among the 55 patients, 15 (all fulfilling the ACR criteria for RA) had MR erosions at baseline; of these, nine had one MR erosion, one had two erosions, two had three, one had four, one had five, and one had seven MR erosions, whereas one from the new-RA group had one erosion. Overall, the median synovial membrane hypertrophy score was 6 (range 0-27) (sum of scores for the eight joints). Figure 1 shows the synovial membrane hypertrophy score distributions of each joint separately and the distribution of swollen or tender joint counts, or both.

\section{Baseline findings for patients followed up for one} year $(n=34)$

Twenty seven erosions were found in 12 patients from the RA group and one erosion was found in a patient from the new-RA group, whereas no erosions were found in the PA group. Figure 2 shows the distribution of MR erosions in each finger joint.

Median synovial membrane hypertrophy scores (sum of scores of the eight joints) were 10 (range 0-25) in the RA group, 4 (range 2-6) in the PA group, and 6 (range 0-12) in the new-RA group. There was a significant difference only between the RA group and the PA group (Mann-Whitney, $\mathrm{p}=0.005$ ) for the synovial membrane hypertrophy score.

Tenosynovitis was found in $17 / 21(81 \%)$ patients in the RA group, in $3 / 5(60 \%)$ patients 


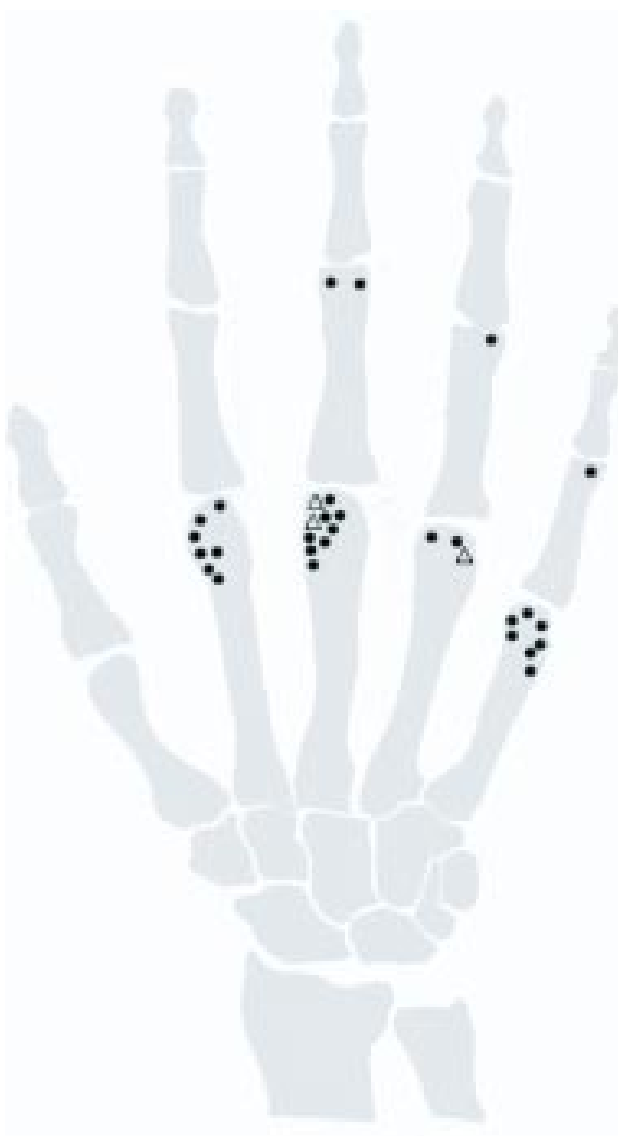

Figure 2 Distribution of magnetic resonance (MR) erosions at baseline and at the one year follow up of the 2 nd to 5th metacarpophalangeal and proximal interphalangeal joints of the dominant hand (of 32 right handed and two left handed patients). A dot represents an MR erosion at baseline. A triangle represents a new $M R$ erosion at the one year follow $u p$.

in the new-RA group, and in $3 / 8$ (38\%) patients in the PA group.

Progression of MR erosions from baseline to one year $(n=34)$

All erosions identified at baseline were also identified at follow up. Two patients without erosions at baseline developed new erosions (one erosion in a patient from the RA group and one in the new-RA group) and one patient with erosions at baseline (RA group) developed one new erosion. Of the three new erosions two occurred in the 3rd MCP joints, and one in the 4 th MCP joint (fig 3). No patient from the PA group developed erosions. No enlargement of erosions was seen.

Change of synovial membrane hypertrophy and tenosynovitis scores from baseline to one year $(n=34)$

In the RA group, the new-RA group, and the PA group no significant differences were found between baseline and one year synovial membrane hypertrophy scores $(\mathrm{p} \geqslant 0.05)$. No significant difference was found between the three groups when the sum of changes in synovial membrane hypertrophy score at baseline and one year were compared (Mann-Whitney, $\mathrm{p}>0.1)$.

Tenosynovitis was less common after one year. Among the 34 patients followed up for one year, tenosynovitis occurred in 23 patients at baseline and in 15 patients at one year (table 2 ). No significant changes within groups were found between baseline and the one year follow up.

CHANGES OVER TIME IN CLINICALLY AFFECTED JOINTS VERSUS CHANGES OVER TIME IN MR DETECTED SYNOVIAL MEMBRANE HYPERTROPHY It appears from table 2 that in the RA and PA groups the synovial membrane hypertrophy scores did not change significantly between baseline and the one year follow up, whereas a significant decrease in the number of swollen and tender joint counts was observed $(\mathrm{p}<0.01)$. In the new-RA group, neither the synovial membrane hypertrophy score nor the clinical assessments of swollen and tender joints changed significantly within the one year observational period.

COMPARISONS OF THE DETECTION OF EROSIONS BY MRI AND RADIOGRAPHY

In the following, assessment of erosions in the 2nd to 5th MCP and PIP joints in the 34 patients followed up for one year will be compared by the two methods.

MRI at baseline versus radiography at baseline ( $n=34$ patients)

Twenty eight erosions were detected by MRI in 13 patients, of which two were detected by radiography. In two patients, radiography detected an erosion which was not seen by MRI, localised in the 3rd and 5th PIP joints, respectively. Thus the ratio of MRI erosions: radiographic erosions was $28: 4=7$.

Progression at one year as assessed by MRI versus radiography ( $n=34$ patients)

MRI detected new erosions in three patients (one in each patient). One of the erosions was detected by radiography at baseline, the two others were not detected by radiography at baseline or at one year.

Radiography only detected two new erosions (both in the same patient) in the MRI scanned area, both of which had already been detected by MRI at baseline (and again at one year).

Radiography detected 24 new erosions in eight patients outside the MRI scanned area. All these patients except one, however, had erosions found in the MR scanned area at baseline (six patients) or at follow up (one patient).

RADIOGRAPHY OF BOTH HANDS AND WRISTS

Baseline findings for all patients $(n=55)$

Fourteen erosions were found in nine patients. The erosions were equally distributed in dominant and non-dominant hands. Three patients had erosions only in the wrist joints, four had erosions only in the hand joints, and two patients had erosions in both wrist and hand joints. The median Larsen score (both hands) of all 55 patients was 0 (range $0-27$ ), as $72 \%$ had Larsen score 0 (mean 3.2, SD 6.5).

Baseline findings of patients followed up for one year $(n=34)$

Erosions were found in six patients. Two patients had erosions only in wrist joints, three 

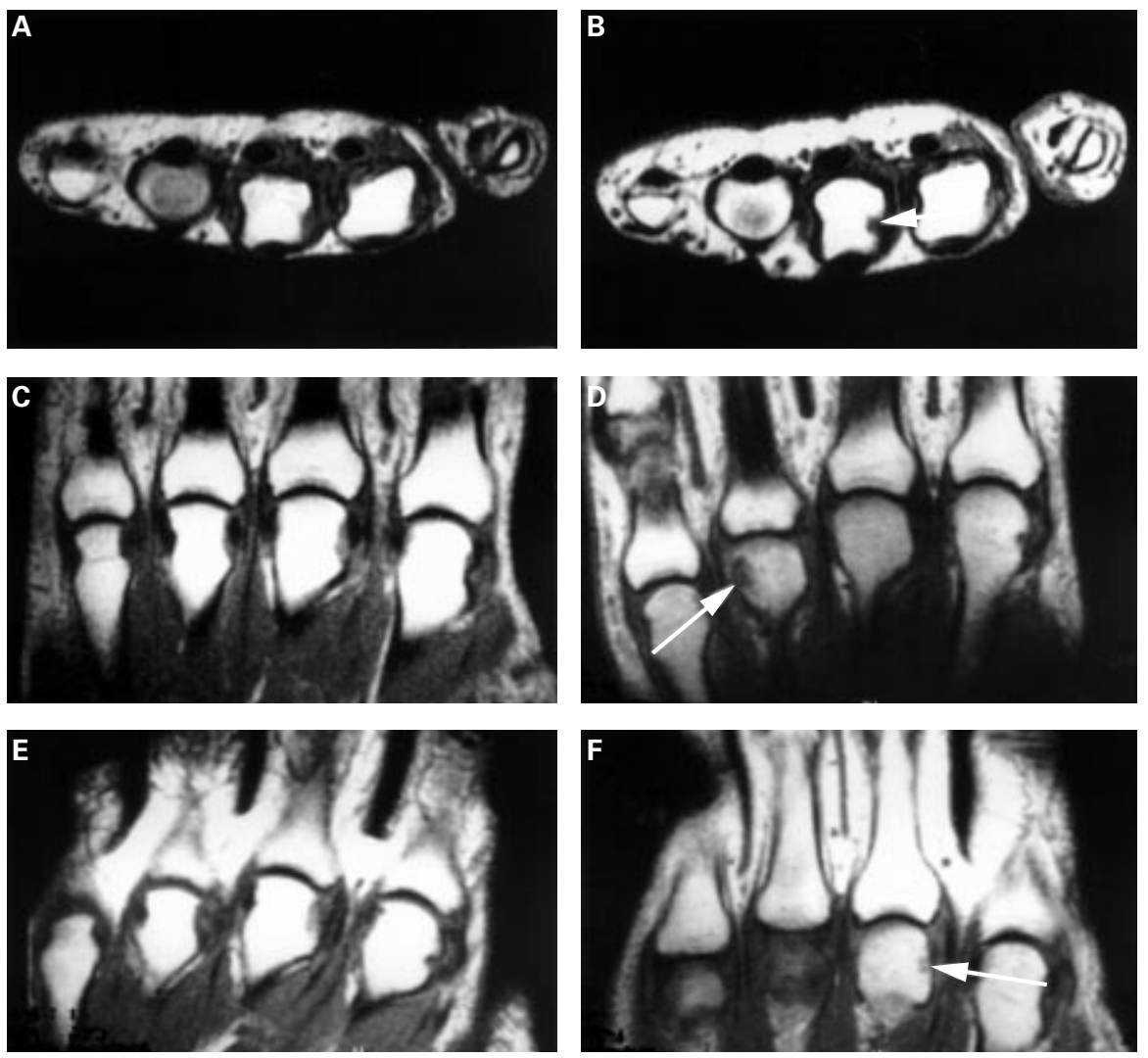

Figure 3 Magnetic resonance images of the three new erosions (white arrows) developed within the observation period of one year in three patients. T1 weighted, spin echo, pre-contrast axial images (A) at baseline, and (B) at the one year follow up; a new erosion is seen in the 3rd metacarpophalangeal (MCP) joint. (C) T1 weighted, spin echo, pre-contrast coronal image at baseline and (D) at the one year follow up, a new erosion is seen in the 4th MCP joint. (E) T1 weighted, spin echo, pre-contrast coronal image at baseline and $(F)$ at the one year follow up, a new erosion is seen in the 3rd MCP joint.

only in the finger joints, and one patient had erosions in both wrist and finger joints (fig 4). The median Larsen score (both hands) of all the patients was 0 (range $0-18$ ), as $74 \%$ had Larsen score 0 (mean 2.4, SD 4.8). The median Larsen scores were 0 (range $0-18$ ) in the RA group (mean 3.3, SD 5.6), 0 (range $0-0$ ) in the PA group (mean 0, SD 0), and 0 (range 0-10) in the new-RA group (mean 2.0, SD 4.5).

Progression from baseline to one year $(n=34)$

All erosions identified at baseline were found again at follow up. Except in two patients, all the new erosions at one year were identified in patients having erosions at baseline. In the wrist joints, 16 new erosions occurred, while in the hand joints, 10 new erosions occurred (sum of scores for both hands) (fig 4). No enlarge- ment of erosions was found. The median Larsen score (both hands) of all the patients was 0 (range $0-29$ ), as $68 \%$ had Larsen score 0 (mean 4.7, SD 8.4). The median Larsen scores were 0 (0-29) in the RA group (significantly different from baseline values, Wilcoxon, $\mathrm{p}=0.007$ ) (mean 7.2, SD 9.8), 0 (range 0-0) in the PA group (NS) (mean $0, \mathrm{SD} 0$ ), and 0 (range 0-10) in the new-RA group (NS) (mean 2.0, SD 4.7).

SCINTIGRAPHY OF THE 2ND TO 5TH MCP AND PIP JOINTS OF THE DOMINANT HAND

At baseline

Twenty four patients were examined. The median sum of scores was 0 (range $0-10$ ), as $63 \%$ had a score of 0 .

Table 2 Sum of scores for the 2nd to the 5th metacarpophalangeal and proximal interphalangeal joints with respect to magnetic resonance imaging (MRI) scores of tenosynovitis and synovial membrane hypertrophy and clinical assessment of swollen and tender joints (34 patients)

\begin{tabular}{|c|c|c|c|c|c|c|c|c|}
\hline & \multicolumn{2}{|c|}{ MRI tenosynovitis } & \multicolumn{2}{|c|}{$\begin{array}{l}\text { MRI synovial membrane } \\
\text { hypertrophy }\end{array}$} & \multicolumn{2}{|c|}{ Swollen joint count } & \multicolumn{2}{|c|}{ Tender joint count } \\
\hline & Baseline & One year & Baseline & One year & Baseline & One year & Baseline & One year \\
\hline RA group $(n=21)$ & $3(0-8)$ & $2.5(0-7)$ & $10(0-25)$ & $12(4-18)$ & $2.5(0-7)$ & $0(0-2)^{\star}$ & $6(0-8)$ & $0(0-8)^{\star \star}$ \\
\hline PA group $(n=8)$ & $0 \quad(0-4)$ & $0 \quad(0-2)$ & $4(2-6)$ & $6(2-10)$ & $1 \quad(0-5)$ & $0(0)^{\star}$ & $6(2-8)$ & $0(0-8)^{\star}$ \\
\hline New-RA group $(n=5)$ & $3.5(0-4)$ & $1 \quad(0-8)$ & $6(0-12)$ & $8(0-15)$ & $2(0-3)$ & $0(0)$ & $5(2-7)$ & $0(0-8)$ \\
\hline
\end{tabular}

${ }^{\star} \mathrm{p}<0.05$ and ${ }^{\star \star} \mathrm{p}<0.001$, significant change from baseline (Wilcoxon matched pairs test) 


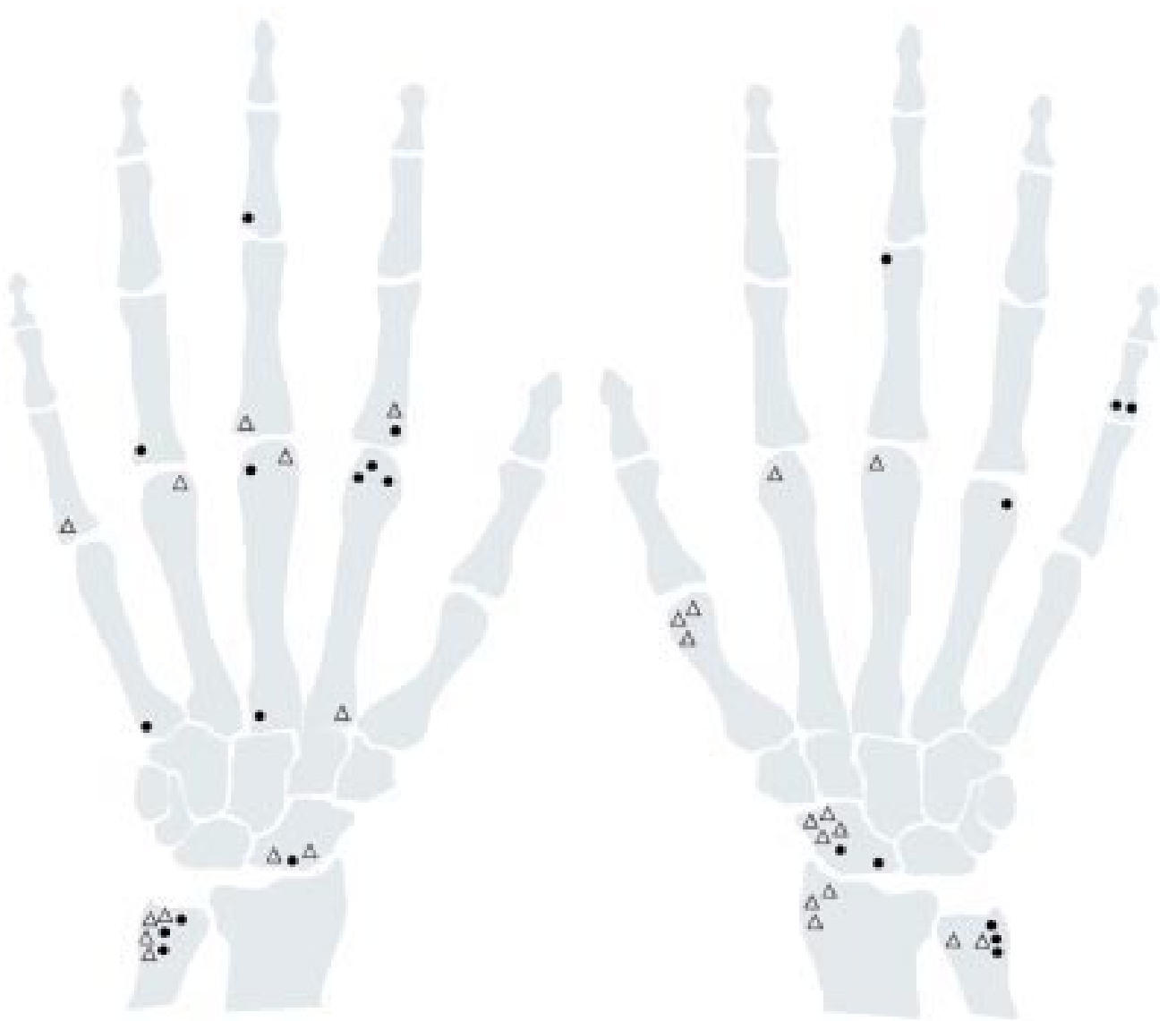

Figure 4 Distribution of radiographic erosions at baseline and at the one year follow up in both hands (34 patients). A dot represents an erosion at baseline. A triangle represents a new erosion at the one year follow up. Erosions found in dominant hands (32 right handed and two left handed) are indicated on the hand to the right in the figure, whereas erosions found in non dominant hands are indicated on the hand to the left.

Progressions from baseline to one year

Fourteen patients (nine from the RA group, two from the PA group, and three from the new-RA group) were followed up for one year. In the 14 patients, baseline results were compared with one year results, showing no significant differences from baseline values within groups or between groups $(\mathrm{p}>0.05)$. In the new-RA and PA groups, applying statistical tests to the scintigraphy data was not meaningful owing to the small number of observations.

COMPARISON OF SCINTIGRAPHY, CLINICAL JOINT ASSESSMENT, AND MRI (14 PATIENTS)

Changes in scintigraphy scores from baseline to the one year follow up did not correlate significantly with MRI synovial membrane hypertrophy scores or MRI erosion scores or clinical assessments of swollen and tender joint counts.

\section{Discussion}

Three subgroups were studied: patients with early RA from the outset (RA group), patients who developed RA over a one year observation period (new-RA group), and patients who remained unclassified (PA group). To our knowledge, this is the first longitudinal MRI study that provides data on the 2 nd to 5 th MCP and PIP joints of patients diagnosed with RA during the observation period. MRI detected erosions at baseline and progression of erosions more often than did radiography.
Furthermore, none of the patients who remained in the PA group developed erosions. The fact that the synovial membrane hypertrophy score of the finger joints did not decrease after the one year follow up, despite reduced clinical signs of synovitis activity, suggests that disease activity may still be present in clinically inactive joints.

If it is assumed that MRI detected erosions represent the same phenomena as the radiographically detected erosions, MRI showed at baseline a larger number of erosions than did radiography of the same finger joints (ratio of MRI erosions to radiographic erosions was $28: 4=7$ at baseline). To confirm that MRI is a more sensitive method than radiography we would expect some MR erosions to develop into radiographically detectable erosions. This occurred only in two cases during the one year follow up.

In this study enlargement of erosions was not seen at follow up. The observation period and the relatively small number of erosions observed may account for this. Another possibility may be that the sensitivity for enlargement of erosions is not sufficiently high. The sensitivity for enlargement of MR erosions is not known and needs to be explored.

MRI detected three new erosions at the follow up. One of the erosions was found in a patient who had not been diagnosed with RA at baseline and who did not develop radiographi- 
cally detectable erosions in either wrist or hand joints, but who fulfilled the ACR 1987 criteria for RA at one year. This example suggests that MRI is more advantageous than radiography. In another patient, however, the new MR erosion had already been seen at baseline by radiography. A possible explanation is that MRI may have overlooked the erosion owing to an unfortunate slice selection of the images or because the slices were $3 \mathrm{~mm}$ thick. In general, the low number of new erosions in our study may reduce the power of the conclusions. From a recent longitudinal study of early RA by Kuper et al, the rate of radiographic progression of erosions can be calculated for individual joint groups (MCP and PIP joints). ${ }^{35}$ Thus among 870 MCP joints and 870 PIP joints studied, 156 and 121 new eroded joints were detected over a three year observation period, respectively. In our study, assuming similar rates of progression (that is, annual rate of progression calculated in the PIP joints = $121 /(870 \times 3) \times 100=4.6 \%)$, we would have expected eight new joints with radiographically detectable erosions at the MCP joints and six joints with new erosions in the PIP joints among the 26 patients diagnosed with RA at one year (RA + new-RA group). We only detected two new erosions in two joints by radiography in the MRI scanned area. These differences by may due to variations between studies in patient characteristics or treatment strategy.

Information about the general disease status of the patients may not be obtained satisfactorily by performing an MRI examination only of the 2nd to 5th MCP and PIP joints of the dominant hand. Thus radiography of both hands and wrists showed more patients with erosive progression. Also, it is noted that progression occurred more often in the wrist joints than in the finger joints. This observation is in accordance with another study, in which a high degree of damage in the wrist was shown. ${ }^{2}$ Additionally, wrist joints are affected more symmetrically than finger joints. ${ }^{36}$ These findings indicate that examining finger joints and wrist joints of both hands in RA may be useful in future assessments of disease activity by MRI.

Two earlier MRI studies have dealt with the predictive value of MR synovial membrane hypertrophy assessments in wrist joints. They showed that MR synovial membrane hypertrophy scores had a high predictive value for the later development of MR erosions in established RA. ${ }^{14}{ }^{37}$ Another, cross sectional study, found a high correlation between bone damage expressed as bone marrow oedema and synovial hypertrophy, indicating that the two phenomena are linked. ${ }^{17}$ In our study, however, MR synovial membrane hypertrophy persisted in several patients without the development of erosions. Our finding may be related to the fact that we studied a group of patients with early disease symptoms, and that some of the patients (the PA group) may be destined not to develop erosions either because the disease was self limiting or because of the early start of treatment. The pathogenesis behind develop- ment of erosions is not fully elucidated, but new theories may help to explain our findings. ${ }^{38}$ Interestingly, animal studies have shown that erosion development may be more closely related to angiogenesis than to swelling of the synovial membrane in the early phase of the disease. ${ }^{38-40}$ Randomised controlled trials monitored with MRI and long term follow up of patients might help to clarify this issue.

In our study the changes in swollen and tender joint counts did not parallel the corresponding changes in synovial membrane hypertrophy assessed by MRI. This is in accordance with other studies ${ }^{14}{ }^{37}$ and agrees with results from other studies that synovial membrane hypertrophy expresses other aspects of disease activity than clinical evaluation. ${ }^{20} 2223$ In particular, more detailed evaluation by MRI may show that pathological conditions within the synovium/synovitis may still be present, although the joints were assessed as clinically inactive.

In many joints only one MR image (slice thickness $3 \mathrm{~mm}$ ) was obtained through the PIP joints. Accordingly, the general impression was that evaluation of the PIP joints was more difficult than evaluation of the MCP joints.

We compared synovial membrane hypertrophy scores and tracer accumulation in the joints by MRI and scintigraphy. The association between tracer uptake and subsequent development of erosions has been studied earlier. ${ }^{41}{ }^{42}$ In contrast with these studies, we did not find markedly increased tracer accumulation in patients with RA as might have been expected. However, it should be emphasised that for the comparisons with MRI we chose only to evaluate the 2 nd to 5 th MCP and PIP joints.

In this study of the symptoms of early arthritis, six patients left the study because of an uneasy or claustrophobic feeling during the MRI examination. These problems may be solved by the use of dedicated MRI scanners, specially designed for imaging the hands and feet. ${ }^{43}$ The large number of dropouts may also be related to the fact that some of the patients found that the examination programme was too time consuming.

In conclusion, in the 2 nd to 5 th $\mathrm{MCP}$ and PIP joints MRI seems to be more sensitive than radiography for the detection of erosions at baseline and for detection of progression of erosions. Patients who continued to have a diagnosis of unclassified polyarthritis at one year did not have erosions at baseline and did not develop erosions, which supports the notion that erosions detected by MRI are the result of a pathological process. Radiography of both hands of the same patients indicated that it might be advantageous to perform MRI of both hands and wrists in early RA and early unclassified polyarthritis. MR synovial membrane hypertrophy and scintigraphy scores did not parallel the changes in the clinical assessment of swollen and tender joint counts. The finding may support the hypothesis that pathological processes may be active in the synovium, despite a lack of clinical signs of synovitis. Long term follow up and larger studies with 
possible histopathological reference are needed to confirm the findings.

We acknowledge the Danish Rheumatism Association, the Thomas and Elisabeth Frølund Nielsen Foundation, Henny og mas and Elisabeth Frølund Nielsen Foundation, Henny og Helge Holgersens Grant, Fonden til Lægevidenskabens Fremme, Emmy Lange, født Kramps Grant, Gårdejer af Stenløse Peder Laurits Pedersens Grant, Oldermand, slagtermester Peter Ryholts Legat, Karen Marie Jørgensen og Datters
Legat, and Lauritz og Augusta Dahl's Mindelegat for financial support. Schering Diagnostika, Denmark is thanked for providing the contrast agent. Nurse Brigitta Pedersen-Zbinden is acknowledged for clinical management of the patients. We thank Susanne Østergaard for skilful photographic assistance.

1 Fleming A, Benn RT, Corbett M, Wood PH. Early rheumatoid disease. II. Patterns of joint involvement. Ann Rheum Dis $1976 ; 35: 361-4$

2 Scott DL, Coulton BL, Popert AJ. Long term progression of joint damage in rheumatoid arthritis. Ann Rheum Dis 1986;45:373-8.

3 Brook A, Corbett M. Radiographic changes in early rheumatoid disease. Ann Rheum Dis 1977;36:71-3.

4 Emery P. Therapeutic approaches for early rheumatoid arthritis. How early? How aggressive? $\mathrm{Br} \mathrm{J}$ Rheumatol 1995;34(suppl 2):87-90.

5 Østergaard M, Gideon P, Sørensen K, Hansen M, Stoltenberg M, Henriksen $\mathrm{O}$, et al. Scoring of synovial membrane hypertrophy and bone erosions by MR imaging membrane hypertrophy and bone erosions by MR imaging in clinically active and inactive rheumatoid

6 McQueen FM, Stewart N, Crabbe J, Robinson E, Yeoman $S$, Tan PL, et al. Magnetic resonance imaging of the wrist in early rheumatoid arthritis reveals a high prevalence of erosions at four months after symptom onset. Ann Rheum Dis 1998;57:350-6.

7 Adam G, Dammer M, Bohndorf K, Christoph R, Fenke F, Gunther RW. Rheumatoid arthritis of the knee: value of gadopentetate dimeglumine-enhanced MR imaging. Am J Roentgenol 1991;156:125-9.

8 Björkengren AG, Geborek P, Rydholm U, Holtas S, Petterson H. MR imaging of the knee in acute rheumatoid arthritis: synovial uptake of gadolinium-DOTA. Am J Roentgenol 1990;155:329-32.

9 Hervé-Somma CM, Sebag GH, Prieur AM, Bonnerot V, Lallemand DP. Juvenile rheumatoid arthritis of the knee: MR evaluation with Gd-DOTA. Radiology 1992;182: MR 8 .

10 Foley-Nolan D, Stack JP, Ryan M, Redmond U, Barry C, Ennis J, et al. Magnetic resonance imaging in the assessment of rheumatoid arthritis - a comparison with plain film radiographs. Br J Rheumatol 1991;30:101-6.

11 Sugimoto H, Takeda A, Masuyama J, Furuse M. Early-stage rheumatoid arthritis: diagnostic accuracy of MR imaging. Radiology 1996;198:185-92.

12 Jevtic V, Watt I, Rozman B, Kos Golja M, Demsar F, Jarh O. Distinctive radiological features of small hand joints in rheumatoid arthritis and seronegative spondyloarthritis demonstrated by contrast-enhanced (Gd-DTPA) magnetic resonance imaging. Skeletal Radiol 1995;24:351-5.

13 Polisson RP, Schoenberg OI, Fischman A, Rubin R, Simon $\mathrm{LS}$, Rosenthal D, et al. Use of magnetic resonance imaging and positron emission tomography in the assessment of synovial volume and glucose metabolism in patients with synovial volume and glucose metabolism in patients with
rheumatoid arthritis. Arthritis Rheum 1995;38:819-25.

14 Østergaard M, Hansen M, Stoltenberg M, Gideon P, Klarlund M, Jensen KE, et al. MRI-determined synovial membrane volume as a marker of disease activity and predictor brane volume as a marker of disease activity and predictor of progressive joint destruction in rheum

15 Sugimoto H, Takeda A, Kano S. Assessment of disease activity in rheumatoid arthritis using magnetic resonance imaging: quantification of pannus volume in the hands. B J Rheumatol 1998;37:854-61.

16 Jevtic V, Watt I, Rozman B, Presetnik M, Logar D, Praprotnik S, et al. Prognostic value of contrast enhanced Gd-DTPA MRI for development of bone erosive changes in rheumatoid arthritis. Br J Rheumatol 1996;35(suppl 3): $26-30$

17 McGonagle D, Conaghan PG, O'Connor P, Gibbon W, Green $M$, Wakefield $\mathrm{R}$, et al. The relationship between synovitis and bone changes in early untreated rheumatoid arthritis: a controlled magnetic resonance imaging study. Arthritis Rheum 1999;42:1706-11.

18 Backhaus M, Kamradt T, Sandrock D, Loreck D, Fritz J, Wolf KJ, et al. Arthritis of the finger joints: a comprehensive approach comparing conventional radiography, scintigraapproach comparing conventional radiography, scintigraphy, ultrasound, and contrast-enhanced magnetic

nance imaging. Arthritis Rheum 1999; 42:1232-45.
19 Klarlund M, Østergaard M, Gideon P, Sørensen K, Jensen $\mathrm{KE}$, Lorenzen I. Wrist and finger joint MR imaging in rheumatoid arthritis. Acta Radiol 1999;40:400-9.

20 Klarlund $M, \varnothing$ stergaard $M$, Lorenzen I. Finger joint synovitis in rheumatoid arthritis. Quantitative assessment by magnetic resonance imaging. Rheumatology 1999;38:6672 .
21 Klarlund M, Østergaard M, Rostrup E, Skjødt H, Lorenzen I. Dynamic magnetic resonance imaging of the metacarpophalangeal joints in rheumatoid arthritis, early unclassified polyarthritis, and healthy controls. Scand J Rheumatol 2000;29:108-15.

22 Jevtic V, Watt I, Rozman B, Kos GM, Praprotnik S, Logar $\mathrm{D}$, et al. Contrast enhanced Gd-DTPA magnetic resonance imaging in the evaluation of rheumatoid arthritis during a clinical trial with DMARDs. A prospective two-year follow-up study on hand joints in 31 patients. Clin Exp Rheumatol 1997;15:151-6.

23 Jevtic V, Rozman B, Watt I, Presetnik M. Use of contrast enhanced MRI in the assessment of therapeutic response to disease-modifying antirheumatic drug. Case study of a patient with rheumatoid arthritis - 6- and 24-month follow up. Br J Rheumatol 1995;34:956-9.

24 Fuchs HA, Pincus T. Radiographic damage in rheumatoid arthritis: description by nonlinear models. J Rheumatol 1992;19:1655-8.

25 Pincus T, Callahan LF, Fuchs HA, Larsen A, Kaye J. Quantitative analysis of hand radiographs in rheumatoid arthritis: time course of radiographic changes, relation to joint examination measures, and comparison of different scoring methods. J Rheumatol 1995;22:1983-9.

26 Larsen A, Thoen J. Hand radiography of 200 patients with rheumatoid arthritis repeated after an interval of one year. Scand J Rheumatol 1987;16:395-401.

27 Salaffi F, Ferraccioli GF. Progress of anatomical damage in rheumatoid hands. Radiography of the natural course of the disease or the course during treatment? Scand J Rheumatol 1989;18:119-20.

28 Wolfe F, Kleinheksel SM, Cathey MA, Hawley DJ, Spitz PW, Fries JF. The clinical value of the Stanford Health Assessment Questionnaire Functional Disability Index in patients with rheumatoid arthritis. J Rheumatol 1988;15: $1480-8$

29 Felson DT, Anderson JJ, Boers M, Bombardier C, Chernoff $\mathrm{M}$, Fried B, et al. The American College of Rheumatology preliminary core set of disease activity measures for rheumatoid arthritis clinical trials. The Committee on Outcome Measures in Rheumatoid Arthritis Clinical Trials. Arthritis Rheum 1993;36:729-40.

30 van der Heijde DM. Plain X-rays in rheumatoid arthritis: overview of scoring methods, their reliability and applicability. Baillieres Clin Rheumatol 1996;10:435-53.

31 Nørgaard F. Earliest roentgenological changes in polyarthritis of the rheumatoid type: rheumatoid arthritis. Radiology 1965;85:325-9.

32 Larsen A, Dale K, Eek M. Radiographic evaluation of rheumatoid arthritis and related conditions by standard reference films. Acta Radiol 1977;18:481-91.

33 Larsen A. How to apply Larsen score in evaluating radiographs of rheumatoid arthritis in long-term studies. J Rheumatol 1995;22:1974-5.

34 Arnett FC, Edworthy SM, Bloch DA, McShane DJ, Fries JF, Cooper NS, et al. The American Rheumatism Association 1987 revised criteria for the classification of rheumatoid arthritis. Arthritis Rheum 1988;31:315-24.

35 Kuper IH, van Leeuwen MA, van Riel PL, Sluiter WJ, Houtman NM, Cats HA, et al. Influence of a ceiling effect on the assessment of radiographic progression in rheumatoid arthritis during the first 6 years of disease. J Rheumatol 1999;26:268-76.

36 Halla JT, Fallahi S, Hardin JG. Small joint involvement: a systematic roentgenographic study in rheumatoid arthritis. Ann Rheum Dis 1986;45:327-30.

37 McQueen FM, Stewart N, Crabbe J, Robinson E, Yeoman $\mathrm{S}$, Tan PL, et al. Magnetic resonance imaging of the wrist in early rheumatoid arthritis reveals progression of erosions despite clinical improvement. Ann Rheum Dis 1999;58: 156-63.

38 Kirwan JR. The relationship between synovitis and erosions in rheumatoid arthritis. Br J Rheumatol 1997;36:225-8.

39 Firestein GS. Starving the synovium: angiogenesis and inflammation in rheumatoid arthritis. J Clin Invest 1999;103:3-4.

40 Storgard CM, Stupack DG, Jonczyk A, Goodman SL, Fox RI, Cheresh DA. Decreased angiogenesis and arthritic disease in rabbits treated with an alpha $\mathrm{v}$ beta3 antagonist. J Clin Invest 1999;103:47-54

41 Möttönen T, Hannonen P, Toivanen J, Rekonen A, Oka M. Value of joint scintigraphy in the prediction of erosiveness in early rheumatoid arthritis. Ann Rheum Dis 1988;47: $183-9$.

42 Van de Wiele C, Van den Bosch F, Mielants H, Simons M, Veys EM, Dierckx RA. Bone scintigraphy of the hands in early stage lupus erythematosus and rheumatoid arthritis. J Rheumatol 1997;24:1916-21.

43 Peterfy CG, Roberts T, Genant HK. Dedicated extremity MR imaging. An emerging technology. Radiol Clin North Am 1997;35:1-20. 